\title{
Scenario Based Global Supply Chain Planning Process Considering Demand Uncertainty
}

\author{
Hongjoo Lee*, Hosang Jung**
}

\begin{abstract}
In this paper, we propose a scenario based global supply chain planning (GSCP) process considering demand uncertainty originated from various global supply chain risks. To generate the global supply chain plan, we first formulate a GSCP model. Then, we need to generate several scenarios which can represent various demand uncertainties. Lastly, a planning procedure for considering those defined scenarios is applied. Unlike the past related researches, we adopt the fuzzy set theory to represent the demand scenarios. Also, a scenario voting process is added to calculate a probability (possibility) of each scenario. An illustrative example based on a real world case is presented to show the feasibility of the proposed planning process.
\end{abstract}

Keywords : Global supply chain planning, Demand uncertainty, Scenario, Fuzzy set theory, Stochastic linear programming

JEL classification number s: C61, L68, M11

* First Author: Assistant Professor, Department of Industrial \& Management Engineering, Kyonggi University, Suwon, Korea.

${ }^{* *}$ Corresponding Author: Assistant Professor, Graduate School of Logistics, Inha University, 100 Inha-ro, Nam-gu, Incheon, Korea. Email: hjung@inha.ac.kr, Phone: 031-860-8435, Fax: 032-860-8226 


\section{INTRODUCTION}

Global supply chain environments are naturally uncertain since various unexpected events can be occurred. Labor strikes, political unstableness, and natural disasters are some of the major risks when trying to generate the exact supply chain plans for upcoming planning periods. In addition to such risks, global manufacturing and sales requiring collaboration with foreign partners make the global supply chain planning environment more complex and uncertain since the customer demands in the foreign markets can be fluctuated and unexpected due to various different reasons (e.g. exchange rate, government policy, etc.).

Demand uncertainty arises from demand volatility or inaccurate demand forecasting, process/production uncertainty may result from the unreliability of the production process due to machine break downs, and supply uncertainty may be caused by poor supplier performance associated with late deliveries or faulty parts (Peidro et al., 2009, Kabak and Ulengin, 2011). Of these three categories, demand uncertainty is the most important because it directly affects production planning and supply schedules over time (Davis, 1993).

Thus, global supply chain planning considering the demand uncertainty has become an important issue.

Especially, according to the practitioners who gave advice and motivation of this research to us, a rough but quick generation of a global supply chain plan considering various scenarios on demand is definitely required for global supply chain planning. This is because all the major contracts with vendors and transporters are usually made by a yearly global production plan.

In this paper, we propose a scenario based global supply chain planning (GSCP) process considering the demand uncertainties originated from various global supply chain risks. To generate the global supply chain plan, we first formulate a GSCP model. Then, we need to generate several scenarios which can represent various demand uncertainties. Lastly, a planning procedure for considering those defined scenarios together is applied.

The paper is organized as follows. Related research works are presented in Section 2. The proposed GSCP model and the related planning procedure are introduced in Section 3. In Section 4, an illustrative example based on a real-world industrial case is presented to show how the proposed model and procedure run. Finally, Section 5 suggests some future research directions with concluding remarks. 


\section{LITERATURE REVIEW}

Supply chain planning is typically applied with two representative approaches, stochastic programming and fuzzy mathematical programming, to manage demand uncertainties. Stochastic programming approaches (e.g. linear programming) that involve uncertainty are particularly suited for problems where data evolve over time and decisions have to be made prior to observing the entire data stream. The most applied and studied stochastic approaches for handling production planning or supply chain planning problems are two-stage stochastic programming models (Zhang et al., 2011). The decision-maker takes some actions in the first stage, after which a random event occurs affecting the outcome of the first-stage decision. A recourse (response) decision can then be taken in the second stage that compensates for any bad effects that might have been experienced as a result of the first-stage decision. In practice, it is common to use scenarios to consider the expected uncertainties in the stochastic programming models (Escudero and Kamesam, 1995). The applications of the two-stage stochastic programming for multi-site production planning or supply chain planning can be found in Leung et al. (2007), Zhang et al. (2011), Karabuk(2008), Shin and Ryu (2010), etc.

As for the fuzzy mathematical programming, various related methodologies have been developed in order to reflect the uncertain aspects of modeling since Zimmermann (1976, 1978 ) introduced the fuzzy set theory into linear programming. In the fuzzy mathematical programming, some of the parameters and/or constraints are set to be the fuzzy numbers (for the parameter) and/or fuzzy equations (for the constraint). Some researchers have tried to apply the fuzzy mathematical programming to supply chain planning problems (Peidro et al., 2009, Selim et al., 2008; Torabi and Hassini, 2009; Jung and Jeong, 2012) so far.

Each approach has pros and cons. If there is an enough amount of historical data regarding demand uncertainties, stochastic approaches can be used to manage upcoming uncertainties in supply chains. However, when historical data are unreliable or unavailable, it may not be the best choice (Wang and Shu, 2005; Pishvaee and Torabi, 2010). Comparing with the stochastic approaches, the fuzzy approaches provide greater computational efficiency and flexibility in fuzzy arithmetic operations (Inuiguchi and Sakawa, 1995; Liang, 2011). Yet, some researchers have pointed out that the fuzzy approaches are based on a too simple assumption about the representation of uncertain values such as triangular or trapezoidal fuzzy numbers (Jung, 2012).

In this paper, we combine two different approaches: stochastic and fuzzy approaches together in order to overcome their weak points and to maximize their strong points. The basic framework of the GSCP is a two-stage stochastic programming model to pursue the 
robustness of solutions (at the second stage, the decision-maker can respond to the unexpected problems occurred after the first stage decisions). Also, the scenarios are generated by using the fuzzy theory to reflect the variability of uncertain values. Unlike the conventional stochastic programming models, the probabilities associated with scenarios are not given, but are determined by the scenario voting of experts.

From Section 3, the detailed descriptions of the proposed GSCP process will be presented.

\section{THE GSCP PROCESS}

\subsection{Model}

In this paper, we adopt one of the existing supply chain planning models because its feasibility and reliability are already validated. Jung and Jeong (2012) proposed a fuzzy linear programming based supply chain planning model before, and we convert this model into our GSCP model by removing its fuzzy properties and changing the objective function from a maximization one to a minimization one.

To formulate a mathematical model, the following assumptions, indices, parameters, and decision variables are required.

\section{Assumptions}

A1: The supply chain is composed of multiple production facilities, multiple distribution centres, and multiple customers.

A2: Products of the production facilities are moved to the customer via distribution centres.

A3: All of the costs (purchasing, production, inventory holding, and transportation), the necessary transportation time, and the production (transportation) capacities are precise and known to the decision-maker.

A4: Planning is for the upcoming several months, and the basic time unit is a month.

The following indices, parameters, and decision variables are required to formulate the model. 


\section{$\underline{\text { Indices }}$}

$f=1, \cdots, F:$ production facility

$d=1, \cdots, D:$ distribution centre

$c=1, \cdots, C$ : customer

$k=1, \cdots, K:$ product

$v=1, \cdots, V:$ vehicle

$t=1, \cdots, T:$ time period (month)

\section{Parameters}

$r_{k f t} \quad$ : unit production cost for product $k$ in production facility $f$ during period $t$

$t_{k f d v t}^{1} \quad$ : cost of transporting one unit of product $k$ from production facility $f$ to distribution centre $d$ using vehicle $v$ during period $t$

$t_{k d c v t}^{2} \quad$ : cost of transporting one unit of product $k$ from distribution centre $d$ to customer $c$ using vehicle $v$ during period $t$

$h_{k f t}^{1} \quad$ : unit inventory holding cost for product $k$ in production facility $f$ during period $t$

$h_{k d t}^{2} \quad$ : unit inventory holding cost for product $k$ in distribution centre $d$ during period $t$

$H_{f t}^{1} \quad$ : inventory capacity of production facility $f$ during period $t$

$H_{d t}^{2} \quad$ : inventory capacity of distribution centre $d$ during period $t$

$s_{k} \quad$ : storage space required for one unit of product $k$

$m_{k f} \quad$ : production capacity required to produce one unit of product $k$ in production facility $f$

$M_{f t} \quad$ : production capacity of production facility $f$ during period $t$

$T_{f d t}^{1} \quad$ : transportation capacity from production facility $f$ to distribution centre $d$ during period $t$

$T_{d c t}^{2} \quad$ : transportation capacity from distribution centre $d$ to customer $c$ during period $t$

$l_{f d v}^{1} \quad:$ transportation time (month) from production facility $f$ to distribution centre $d$ using vehicle $v$

$l_{d c v}^{2} \quad$ : transportation time (month) from distribution centre $d$ to customer $c$ using vehicle $v$

$\widetilde{D_{k c t}} \quad:$ (uncertain) customer demand for product $k$ by customer $c$ during period $t$ 


\section{Decision Variables}

$x_{k f t} \quad$ : amount of product $k$ produced in production facility $f$ during period $t$

$y_{k f d v t}^{1} \quad$ : amount of product $k$ transported from production facility $f$ to distribution centre $d$ using vehicle $v$ during period $t$

$y_{k d c v t}^{2} \quad$ : amount of product $k$ transported from distribution centre $d$ to customer $c$ using vehicle $v$ during period $t$

$I_{k f t}^{1} \quad$ : inventory amount of product $k$ in production facility $f$ during period $t$

$I_{k d t}^{2} \quad$ : inventory amount of product $k$ in distribution centre $d$ during period $t$

Using the above defined notations, the GSCP model can be formulated as follows:

\section{GSCP model}

$$
\operatorname{Min} \sum_{k=1}^{K} \sum_{f=1}^{F} \sum_{t=1}^{T}\left(r_{k f t} x_{k f t}+h_{k f t}^{1} I_{k f t}^{1}\right)+\sum_{k=1}^{K} \sum_{d=1}^{D} \sum_{t=1}^{T} h_{k d t}^{2} I_{k d t}^{2}+\sum_{k=1}^{K} \sum_{f=1}^{F} \sum_{d=1}^{D} \sum_{v=1}^{V} \sum_{t=1}^{T} t_{k f d v t}^{1} y_{k f d v t}^{1}+\sum_{k=1}^{K} \sum_{d=1}^{D} \sum_{c=1}^{C} \sum_{v=1}^{V} \sum_{t=1}^{T} t_{k d c v t}^{2} y_{k d c v t}^{2}
$$

\section{subject to}

$$
\begin{array}{ll}
I_{k f t}^{1}=I_{k f t-1}^{1}+x_{k f t}-\sum_{d=1}^{D} \sum_{v=1}^{V} y_{k f d v t}^{1} & \forall k, f, t \\
I_{k d t}^{2}=I_{k d t-1}^{2}+\sum_{f=1}^{F} \sum_{v=1}^{V} y_{k f d v t-l_{f d v}^{1}}^{1}-\sum_{c=1}^{C} \sum_{v=1}^{V} y_{k d c v t}^{2} & \forall k, d, t \\
\sum_{d=1}^{D} \sum_{v=1}^{V} y_{k d c v t-l_{d c v}^{2}}^{2}=\widetilde{D}_{k c t} & \forall k, c, t \\
\sum_{k=1}^{K} m_{k f} x_{k f t} \leq M_{f t} & \forall f, t \\
\sum_{k=1}^{K} s_{k} I_{k f t}^{1} \leq H_{f t}^{1} & \forall f, t \\
\sum_{k=1}^{K} s_{k} I_{k d t}^{2} \leq H_{d t}^{2} & \\
\sum_{k=1}^{K} \sum_{v=1}^{V} s_{k} y_{k f d v t}^{1} \leq T_{f d t}^{1} & \forall d, t
\end{array}
$$




$$
\begin{array}{ll}
\sum_{k=1}^{K} \sum_{v=1}^{V} s_{k} y_{k d c v t}^{2} \leq T_{d c t}^{2} & \forall d, c, t \\
I_{k f 0}^{1}=0 \quad, \quad I_{k d 0}^{2}=0 & \forall k, f, d \\
x_{k f t}, I_{k f t}^{1}, I_{k d t}^{2}, y_{k f d v t}^{1}, y_{k d c v t}^{2} \geq 0 & \forall k, f, d, c, v, t
\end{array}
$$

The objective function (1) works to minimize total costs. Constraint (2) models inventory balances for production facilities. Constraint (3) is the flow conservation equation for product transfer between production facilities and customers by way of distribution centres. Constraint (4) ensures that the demand should be fulfilled. Constraint (5) ensures that production capacity is not violated. Constraints (6) and (7) address the inventory capacities at the production facility and the distribution centre, respectively. Constraints (8) and (9) limit the amount of transportation along each transportation route to its transportation capacity. Constraint (10) initializes the inventories of the production facility and the distribution centre to zero to prevents errors in Constraints (2) and (3). Finally, Constraint (11) requires that values are non-negative.

\subsection{Scenario generation on demand uncertainty}

To consider the demand uncertainty in our GSCP model, we develop scenarios which represent various possible conditions of demand. In this paper, the customer demand To consider supply and demand uncertainties in our GSCP model, we develop scenarios which represent various possible conditions of demand. In this paper, the customer demand $\left(D_{k c t}\right)$ is a parameter showing the demand in supply chains.

Even if several different scenarios can be developed, we assume that there exist three scenarios: pessimistic, neutral, and optimistic for demand. Also, in this paper, each scenario is built up using a fuzzy number in order to consider the variability of demand.

For example, a triangular fuzzy number can be used to represent each scenario for demand. A triangular fuzzy number $\tilde{a}$ can be defined by the degree of membership $\left(\mu_{\tilde{a}}(x)\right)$ of element $x$ in the fuzzy set of $a$ using the closed interval $[l, u]$ including its mean $m$ as follows:

$$
\mu_{\tilde{a}}(x)=\left\{\begin{array}{c}
\frac{x-l}{m-l}, l \leq x \leq m, \\
\frac{u-x}{u-m}, m \leq x \leq u, \\
0, \text { otherwise }
\end{array}\right.
$$


Also, the triangular fuzzy number $\tilde{a}$ can be characterized by defining the interval of confidence level $\alpha$ as follows:

$$
\widetilde{a^{\alpha}}=\left\lfloor l^{\alpha}, u^{\alpha}\right\rfloor=[(m-l) \alpha+l, u-(u-m) \alpha] \quad \forall \alpha \in[0,1]
$$

This is $\alpha$-cut method that is known to incorporate the experts or decision-makers confidence over his/her preference or the judgments (Güngör et al., 2009). As shown in Equation (13), the $\alpha$-cut method yields an interval set of values from a fuzzy number: the lower limit and upper limit of the fuzzy numbers with respect to $\alpha$-cut. In general, the value of $\alpha$ is set between 0 and 1 . If $\alpha=0$, it indicates that the degree of uncertainty is greatest and the degree of confidence is least. If $\alpha$ is near to 1 , the degree of uncertainty decreases and the degree of confidence increases (Pan, 2008).

For example, if the pessimistic scenario of demand is given as the triangular fuzzy number, 1000, this can be depicted as shown in Figure 1.

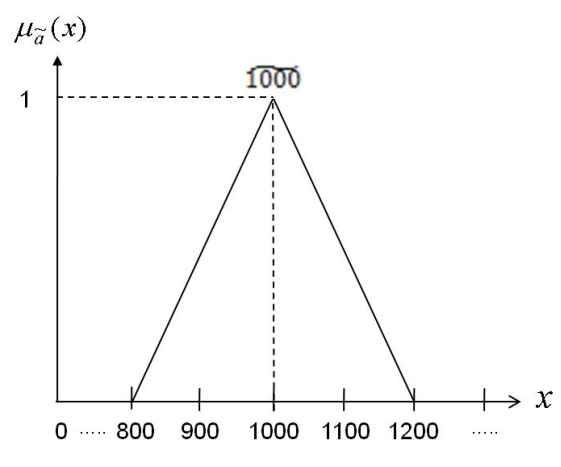

Figure 1.

Example of a triangular fuzzy number

Similarly, the neutral or optimistic scenario can also be represented using the triangular fuzzy number.

Once the scenario is represented as the triangular fuzzy number, we need to convert it into a crisp value. This can be done by applying the index of optimism, $\mu$, which also represents the attitude of the decision-maker (Vahidnia et al., 2009). A larger value of $\mu$ indicates a higher degree of optimism (Ayağ and Özdemir, 2007). The index of optimism is a linear convex combination as follows (Lee, 1999):

$$
C\left(\widetilde{a_{i j}^{\alpha}}\right)=\mu a_{i j u}+(1-\mu) a_{i j l} \quad \forall \mu \in[0,1]
$$


where $C\left(\widetilde{a_{i j}^{a}}\right)$ is the crisp value corresponding to $\widetilde{a_{i j}^{\alpha}}$ considering the index of optimism $\mu$.

Finally, through the converting process of the fuzzy number, three scenarios with the crisp values can be obtained for demand.

\subsection{Scenario voting}

After constructing the scenario, the survey for scenario voting is conducted to calculate its possibility. The calculated possibility of each scenario will be considered when running the GSCP model in the following sub section.

For example, 10 demand forecasting experts might be asked to answer the following question: do you think the pessimistic demand scenario for product $k=1$ during time period 2 will be realized? The number of positive (yes) responses from the experts can be the possibility of that scenario. The forecasting experts can provide multiple positive responses to the scenarios.

Let us suppose that the possibilities of pessimistic, neutral, and optimistic scenarios for the upcoming demand are $0.3,0.5$, and 0.6 . In this case, the final possibility of three scenarios for the demand will be $0.21(=0.3 / 1.4), 0.36(=0.5 / 1.4)$, and $0.43(=0.6 / 1.4)$.

\subsection{Scenario based GSCP}

With the formulated mathematical model, the scenarios on demand, and the final possibilities of scenarios, we can run the scenario based GSCP.

As briefly explained in Section 2, the two-stage stochastic programming model is used to consider the supply and demand uncertainties for GSCP.

Decisions that are implemented before are known as first-stage decisions while those after are second-stage decisions. The first-stage decision variables can be regarded as proactive and are often associated with planning issues, and the second-stage decisions allow the decision-makers to model a response to the observed outcome, which constitutes the recourse (Sen and Higle, 1999). Among several (recourse) polices for the two-stage stochastic programming model, we select a simple recourse policy that imposes a penalty cost when any random parameter such as demand deviates from prescribed limits. For example, when supply chain planning, the first-stage decisions might correspond to production amounts, and demand might be randomly modeled. When demand exceeds the production amounts, this policy may dictate that demand be backlogged or lost sales at some penalty costs. 
Sen and Higle (1999) provided a generic two-stage formulation under a simple recourse policy as follows:

$\operatorname{Min} c x+\sum_{i \in R} g_{i} E\left[\left|\tilde{b}_{i}-\tilde{a}_{i} x\right|\right]$

subject to

$A_{1} x=b_{1}$

$L_{1} \leq x \leq U_{1}$

In equations (15) and (16), let us suppose that $A_{1}$ is a sub-matrix of $A$ and $b_{1}$ is a sub-vector of $b$ which contain only deterministic parameters, when the rows of a deterministic linear programming are written as $A x=b$. We can refer to this portion of the problem as the deterministic part. The remaining rows (containing at least one random element) will be indexed by the set $R$. We refer to $a_{1}$ as the th row vector in $A$, and use $\tilde{a}$ to reflect the presence of random parameters. Also, let $g_{1}>0$ denote the penalty cost for violating the target $\tilde{b}_{i}$.

Whenever the random vectors $\left\{\left(\tilde{a}_{i}, \tilde{b}_{i}\right)\right\}_{i \in R}$ are discrete random variables, the above formulation can be rewritten as a linear program as follows.

$\operatorname{Min} c x+\sum_{i \in R} g_{i}\left(\sum_{s \in S_{i}} p_{i s}\left(Z_{i s}^{+}+Z_{i s}^{-}\right)\right)$

subject to

$$
\begin{aligned}
& A_{1} x+b_{1} \\
& a_{i s} x+z_{i s}^{+}-z_{i s}^{-}=b_{i s} \\
& L_{1} \leq x \leq U_{1} \\
& z_{i s}^{+}, z_{i s}^{-} \geq 0
\end{aligned}
$$

In equations (18)-(20), $S_{i}$ denote an index set of all scenarios regarding the random vector $\left\{\left(\widetilde{a_{i}}, \tilde{b}_{i}\right)\right\}$ and $p_{i s}=P\left\{\left(\tilde{a}_{i}, \tilde{b}_{i}\right)=\left(a_{i s}, b_{i s}\right)\right\}$.

While the simple recourse policy offers a notion of feasibility for first-stage plans, the recourse actions themselves are quite limited. For example, in a production-and-inventory system that is experiencing shortages, a simple recourse policy is one that simply allows the manufacturer to adopt an outsourcing option. A more general recourse policy would allow 
changes in production rates, thus allowing greater flexibility (Sen and Higle, 1999).

Thus, using the above formulation approach, we can convert the GSCP model into the scenario based GSCP (S-GSCP) model as follows:

\section{$\underline{\text { S-GSCP model }}$}

$$
\begin{aligned}
& \operatorname{Min} \sum_{k=1}^{K} \sum_{f=1}^{F} \sum_{t=1}^{T}\left(r_{k f t} x_{k f t}+h_{k f t}^{1} I_{k f t}^{1}\right)+\sum_{k=1}^{K} \sum_{d=1}^{D} \sum_{t=1}^{T} h_{k d t}^{2} I_{k d t}^{2}+\sum_{k=1}^{K} \sum_{f=1}^{F} \sum_{d=1}^{D} \sum_{v=1}^{V} \sum_{t=1}^{T} t_{k f d v t}^{1} y_{k f d v t}^{1}+\sum_{k=1}^{K} \sum_{d=1}^{D} \sum_{c=1}^{C} \sum_{v=1}^{V} \sum_{t=1}^{T} t_{k d c v t}^{2} y_{k d c v t}^{2} \\
& +g\left\{p_{o} \sum_{k=1}^{K} \sum_{c=1}^{C} \sum_{t=1}^{T}\left(z_{k c t}^{o+}+z_{k c t}^{o-}\right)+p_{n} \sum_{k=1}^{K} \sum_{c=1}^{C} \sum_{t=1}^{T}\left(z_{k c t}^{n+}+z_{k c t}^{n-}\right)+p_{p} \sum_{k=1}^{K} \sum_{c=1}^{C} \sum_{t=1}^{T}\left(z_{k c t}^{p+}+z_{k c t}^{p-}\right)\right\}
\end{aligned}
$$

subject to

$$
\begin{aligned}
& I_{k f t}^{1}=I_{k f t-1}^{1}+x_{k f t}-\sum_{d=1}^{D} \sum_{v=1}^{V} y_{k f d v t}^{1} \quad \forall k, f, t \\
& I_{k d t}^{2}=I_{k d t-1}^{2}+\sum_{f=1}^{F} \sum_{v=1}^{V} y_{k f d v t-l_{f d v}^{1}}^{1}-\sum_{c=1}^{C} \sum_{v=1}^{V} y_{k d c v t}^{2} \quad \forall k, d, t \\
& \sum_{d=1}^{D} \sum_{v=1}^{V} y_{k d c v t-l_{d c v}^{2}}^{2}+z_{k c t}^{o+}-z_{k c t}^{o-}=D_{k c t}^{o p t i m i s t i c} \quad \forall k, c, t \\
& \sum_{d=1}^{D} \sum_{v=1}^{V} y_{k d c v t-l_{d c v}^{2}}^{2}+z_{k c t}^{n+}-z_{k c t}^{n-}=D_{k c t}^{n e u t r a l} \quad \forall k, c, t \\
& \sum_{d=1}^{D} \sum_{v=1}^{V} y_{k d c v t-l_{d c v}^{2}}^{2}+z_{k c t}^{p+}-z_{k c t}^{p-}=D_{k c t}^{\text {pessimistic }} \quad \forall k, c, t \\
& \sum_{k=1}^{K} m_{k f} x_{k f t} \leq M_{f t} \\
& \forall f, t \\
& \sum_{k=1}^{K} s_{k} I_{k f t}^{1} \leq H_{f t}^{1} \quad \forall f, t \\
& \sum_{k=1}^{K} s_{k} I_{k d t}^{2} \leq H_{d t}^{2} \quad \forall d, t \\
& \sum_{k=1}^{K} \sum_{v=1}^{V} s_{k} y_{k f d v t}^{1} \leq T_{f d t}^{1} \quad \forall f, d, t \\
& \sum_{k=1}^{K} \sum_{v=1}^{V} s_{k} y_{k d c v t}^{2} \leq T_{d c t}^{2} \quad \forall d, c, t \\
& I_{k f 0}^{1}=0, I_{k d 0}^{2}=0 \\
& \forall k, f, d \\
& x_{k f t}, I_{k f t}^{1}, I_{k d t}^{2}, y_{k f d v t}^{1}, y_{k d c v t}^{2} \geq 0 \quad \forall k, f, d, c, v, t \\
& z_{k c t}^{o+}, z_{k c t}^{o-}, z_{k c t}^{n+}, z_{k c t}^{n-}, z_{k c t}^{p+}, z_{k c t}^{p-} \geq 0 \quad \forall k, c, t
\end{aligned}
$$


In the objective function (23), $g$ is the penalty cost for violating the optimistic, neutral, and pessimistic scenarios on customer demand. Also, $p_{o}, p_{n}$, and $p_{p}$ are the probabilities of three scenarios, respectively. As shown in equations (26)-(28), there are three different scenarios on customer demand: $D_{k c t}^{\text {optimistic }}, D_{k c t}^{\text {neutral }}$, and $D_{k c t}^{\text {pessimistic }}$.

The procedure of the proposed S-GSCP can be depicted as shown in Figure 2.

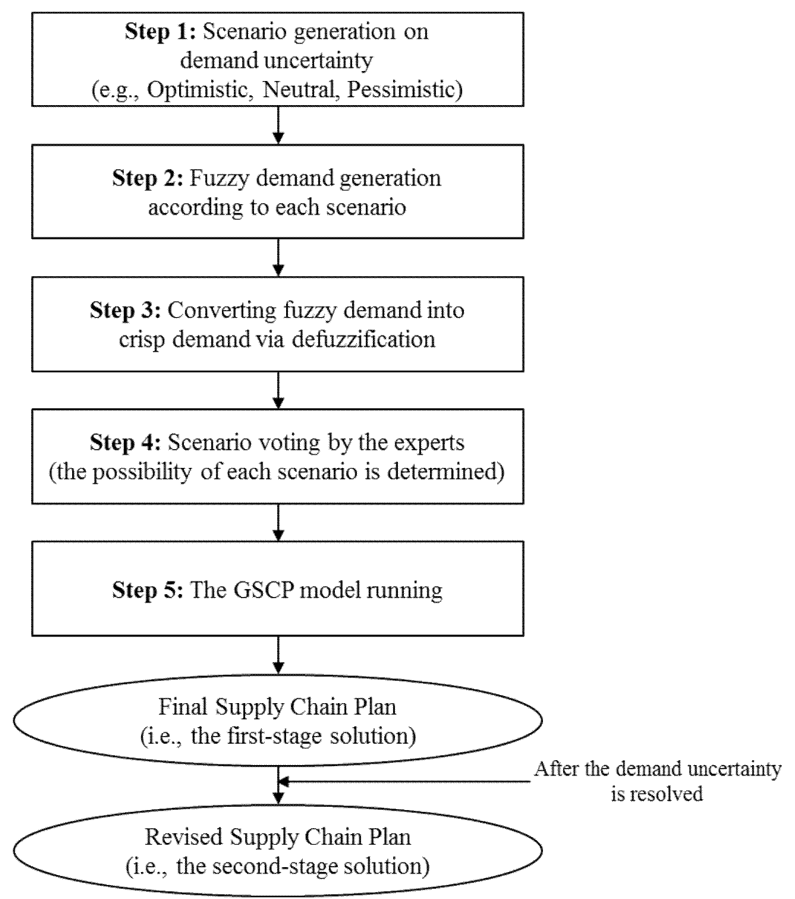

Figure 2.

Overall procedure of the S-GSCP

\section{ILLUSTRATIVE EXAMPLE}

To illustrate the proposed scenario based GSCP, we adopt a real industrial case with data from Jung and Jeong (2012). The adopted case is about Company A, a South Korean electronics company that produces smart phones and home appliances. To simplify the example, only domestic sale network is considered despite the fact Company A has a global sales network.

Except for the uncertain demand and the manufacturing capacity (i.e. supply), the 
summary of the experimental data for this illustrative example is as shown in Table 1.

Table 1.

Summary of the experimental data (Jung and Jeong, 2012)

\begin{tabular}{|c|c|}
\hline Parameters & Values (for $\forall t=1, \ldots, 6$ ) \\
\hline $\begin{array}{l}\text { \# of production facilities }(f) \\
\text { \# of distribution centers }(d)\end{array}$ & 2 \\
\hline $\begin{array}{c}\text { \# of customers }(c) \text {,\#ofproducts }(k) \\
\text { \# of vehicle types }(v)\end{array}$ & 1 \\
\hline Planning time $(t)$ & 6 months \\
\hline Production cost $\left(r_{k f t}\right)$ & $\$ 126$ for $f=1, \$ 128$ for $f=2$ \\
\hline Transportation cost $\left(t_{k f d v t}^{1}\right)$ & $\begin{array}{l}\$ 5 \text { between } f=1 \text { and } d=1 \\
\$ 6 \text { between } f=1 \text { and } d=2 \\
\$ 4 \text { between } f=2 \text { and } d=1 \\
\$ 7 \text { between } f=2 \text { and } d=2\end{array}$ \\
\hline Transportation cost $\left(t_{k d c v t}^{2}\right)$ & $\begin{array}{l}\$ 10 \text { between } d=1 \text { and } c=1 \\
\$ 12 \text { between } d=2 \text { and } c=1\end{array}$ \\
\hline Inventory holding cost $\left(h_{k f t}^{1}\right)$ & $\$ 4$ for $f=1, \$ 2$ for $f=2$ \\
\hline Inventory holding cost $\left(h_{k d t}^{2}\right)$ & $\$ 8$ for $d=1, \$ 9$ for $d=2$ \\
\hline Penalty cost $(g)$ & $\$ 10000$ \\
\hline Inventory capacity $\left(H_{f t}^{1}\right)$ & 5000 for $f=1,4500$ for $f=2$ \\
\hline Inventory capacity $\left(H_{d t}^{2}\right)$ & 15000 for $d=1,13000$ for $d=2$ \\
\hline Storage space $\left(s_{k}\right)$ & 1 \\
\hline Manufacturing capacity $\left(M_{f t}\right)$ & 28000 for $f=1,17000$ for $f=2$ \\
\hline $\begin{array}{l}\text { Production capacity required to produce one unit } \\
\text { of product }\left(m_{k f}\right)\end{array}$ & 1 for $f=1,1$ for $f=2$ \\
\hline Transportation capacity $\left(T_{f d t}^{1}\right)$ & $\begin{array}{l}8500 \text { between } f=1 \text { and } d=1 \\
6500 \text { between } f=1 \text { and } d=2 \\
5500 \text { between } f=2 \text { and } d=1 \\
3000 \text { between } f=2 \text { and } d=2\end{array}$ \\
\hline Transportation capacity $\left(T_{d c t}^{2}\right)$ & $\begin{array}{l}15000 \text { between } d=1 \text { and } c=1 \\
10000 \text { between } d=2 \text { and } c=1\end{array}$ \\
\hline
\end{tabular}


In addition, the customer demand will be determined by the scenario generation process in the following sub section.

\subsection{Scenario generation for the example}

As mentioned in Section 3.2, we assume that there are three different scenarios on demand $\left(D_{k c t}\right)$ : pessimistic, neutral, and optimistic. Based on the given data from Jung and Jeong (2012), we generate the scenarios as shown in Table 2.

Table 2.

Scenarios on the fuzzy demand

\begin{tabular}{|c|c|c|c|c|c|c|}
\hline Demand (unit: 1000) & $t=1$ & $t=2$ & $t=3$ & $t=4$ & $t=5$ & $t=6$ \\
\hline$D_{\text {kct }}^{\text {optimistic }}$ & {$[18,20]$} & {$[19,21]$} & {$[20,22]$} & {$[18,20]$} & {$[17,19]$} & {$[20,22]$} \\
\hline$D_{\text {kcut }}^{\text {neutral }}$ & {$[12,14]$} & {$[13,15]$} & {$[14,16]$} & {$[12,14]$} & {$[11,13]$} & {$[14,16]$} \\
\hline$D_{\text {kct }}^{\text {pessimistic }}$ & {$[6,8]$} & {$[7,9]$} & {$[8,10]$} & {$[6,8]$} & {$[5,7]$} & {$[8,10]$} \\
\hline
\end{tabular}

In Table 2, all the values are represented as the triangular fuzzy numbers using equation (13). Thus, using the equation (14), we need to convert those fuzzy numbers into the crisp values. In this example, $\alpha$ (confidence level on the judgments (or forecasts) of the decision-maker) was set to 1 and $\mu$ (optimism index) was set to 0.5 . Table 3 shows the converted crisp values for the demand.

Table 3.

Crisp values for the demand

\begin{tabular}{|c|c|c|c|c|c|c|}
\hline Demand (unit: 1000) & $t=1$ & $t=2$ & $t=3$ & $t=4$ & $t=5$ & $t=6$ \\
\hline$D_{\text {kct }}^{\text {optimistic }}$ & 19 & 20 & 21 & 19 & 18 & 21 \\
\hline$D_{\text {kct }}^{\text {neutral }}$ & 13 & 14 & 15 & 13 & 12 & 15 \\
\hline$D_{\text {kct }}^{\text {pessimistic }}$ & 7 & 8 & 9 & 7 & 6 & 9 \\
\hline
\end{tabular}

\subsection{Scenario voting for the example}

To calculate the probability (possibility) of each scenario, we asked five senior research engineers working for the production system department of Company A to answer the questions (e.g. do you think the optimistic demand scenario for product $k=1$ during 
time period 1 will be realized?). As explained in Section 3.3, the number of positive (yes) responses from them was utilized to calculate the probability of the scenario. As for the three scenarios, 4,3 , and 1 research engineers gave a positive (yes) response. Thus, the final probabilities of three demand scenarios: $p_{o}, p_{n}$, and $p_{p}$ are $0.5(=0.8 / 1.6), 0.375(=0.6 / 1.6)$, and $0.125(=0.2 / 1.6)$, respectively.

\subsection{S-GSCP model running}

Finally, we generated a supply chain plan for Company A for the upcoming six months by solving the S-GSCP model with IBM ILOG CPLEX Optimization Studio, version 12.5 . The objective function value was $\$ 236,597,000$, and a summary of the supply chain plan is presented in Table 4. The computing execution time was under 1 second.

\section{Table 4.}

Summary of the supply chain plan

\begin{tabular}{|c|c|}
\hline Variables & Values \\
\hline $\begin{array}{c}\text { Production } \\
\text { amount }\left(x_{k f t}\right)\end{array}$ & $\begin{array}{r}f=1: 8500(t=1), 8500(t=2), 9500(t=3), \\
8500(t=4), 8500(t=5), 9500(t=6), \\
f=2: 4500(t=1), 5500(t=2), 5500(t=3), \\
4500(t=4), 3500(t=5), 5500(t=6)\end{array}$ \\
\hline $\begin{array}{l}\text { Transportation amount between the } \\
\text { production facility and the } \\
\text { distribution centre }\left(t_{k f d v t}^{1}\right)\end{array}$ & $\begin{array}{l}\text { From } f=1 \text { to } d=1: 8500(t=1), 8500(t=2), \\
\quad 8500(t=3), 8500(t=4), 8500(t=5), 8500(t=6) \\
\text { From } f=1 \text { to } d=2: 0(t=1), 720(t=2), \\
\quad 1000(t=3), 0(t=4), 0(t=5), 1000(t=6) \\
\text { From } f=2 \text { to } d=1: 4500(t=1), 5500(t=2), \\
\quad 5500(t=3), 4500(t=4), 3500(t=5), 5500(t=6) \\
\text { From } f=2 \text { to } d=2: 0(t=1), 0(t=2), 0(t=3), \\
\quad 0(t=4), 0(t=5), 0(t=6)\end{array}$ \\
\hline $\begin{array}{l}\text { Transportation } \\
\text { amount between the distribution } \\
\text { centre and the customer }\left(t_{k d c v t}^{2}\right)\end{array}$ & $\begin{array}{l}\text { From } d=1 \text { to } c=1: 13000(t=1), 14000(t=2) \\
\quad 14000(t=3), 13000(t=4), 12000(t=5) \\
\quad 14000(t=6) \\
\text { From } d=2 \text { to } c=1: 0(t=1), 0(t=2), 1000(t=3), \\
\quad 0(t=4), 0(t=5), 1000(t=6)\end{array}$ \\
\hline$z_{k c t}^{o+}, z_{k c t}^{o-}, z_{k c t}^{n+}, z_{k c t}^{n-}, z_{k c t}^{p+}, z_{k c t}^{p-}$ & 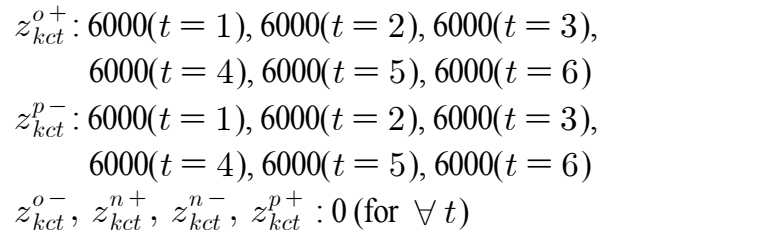 \\
\hline
\end{tabular}


As shown in Table 4, the solution tries to fulfill the neutral demand in order to reduce the penalty costs as much as possible, and this leads to either oversupply $\left(z_{k c t}^{p-}>0\right)$ for the pessimistic demand scenario or undersupply $\left(z_{k c t}^{o+}>0\right)$ for the optimistic demand scenario. Of course, the solution will be changed according to the setting of the probabilities of the scenarios.

The solution of Table 4 can be regarded as the first-stage solution. After the uncertainty of demand is resolved (i.e. one of three scenarios is fixed), a recourse (response) decision will be able to be taken in the second stage that compensates for any bad effects that might have been experienced as a result of the first-stage decision. As mentioned earlier, simply adopting an outsourcing strategy or allowing changes in production rates can be a good option.

\section{CONCLUSIONS}

In this paper, we propose a scenario based global supply chain planning (GSCP) process considering demand uncertainty originated from various global supply chain risks. To generate the global supply chain plan, we first formulated the S-GSCP model. Then, we developed several scenarios which can represent various demand uncertainty and the probabilities of the scenarios. Lastly, a final supply chain plan was generated by considering the predefined scenarios and their probabilities together. Unlike the past related researches, we combined two different approaches: stochastic and fuzzy approaches together in order to overcome their weak points and to maximize their strong points. The proposed S-GSCP model was based on the two-stage stochastic programming model with a simple recourse policy to pursue the robustness the solutions. The uncertain demands in scenarios were represented as the fuzzy numbers to allow variability. Also, the probability of each scenario was determined by the scenario voting process. To show the feasibility of the proposed S-GSCP process, the illustrative example based on a real world case was presented. For future research, we need to perform sensitivity analysis with various combinations of the probabilities of three scenarios. In addition, we need to consider the more various scenarios on supply chain uncertainty such as manufacturing and transportation uncertainties when running the S-GSCP model. Also, if the number of scenario increases, the complexity regarding the scenario reduction might become a serious problem when applying the proposed planning process to the real world situations. Thus, the proper scenario handling techniques need to be developed. 


\section{ACKNOWLEDGEMENTS}

This research was supported by Basic Science Research Program through the National Research Foundation of Korea (NRF) funded by the Ministry of Education, Science and Technology (NRF-2012R1A1A1011396). 


\section{REFERENCES}

Ayağ, Z. and Özdemir, R. G. (2007) An intelligent approach to ERP software selection through fuzzy ANP, International Journal of Production Research, 45(10), 2169-2194.

Davis, T. (1993) Effective supply chain management, Sloan Management Review, 34, 35-46.

Escudero, L. F. and Kamesam, P. (1995) On solving stochastic production planning problems via scenario modeling, TOP, 3(1), 69-95.

Güngör, Z., Serhadlıoğlu, G. and Kesen, S. E. (2009) A fuzzy AHP approach to personnel selection problem, Applied Soft Computing, 9, 641-646.

Inuiguchi, M. and Sakawa, M. (1995) A possibilistic linear programming program in equivalent to a stochastic linear program in special case, Fuzzy Sets and Systems, 76, 309-318.

Jung, H. (2012) An available-to-promise process considering production and transportation uncertainties and multiple performance measures, International Journal of Production Research, 50(7), 1780-1798.

Jung, H. and Jeong, S.-J. (2012) Managing demand uncertainty through fuzzy inference in supply chain planning, International Journal of Production Research, 50(19), 5415-5429.

Kabak, O. and Ulengin, F. (2011) Possibilistic linear-programming approach for supply chain networking decisions, European Journal of Operational Research, 209, 253-264.

Karabuk, S. (2008) Production planning under uncertainty in textile manufacturing, Journal of the Operational Research Society, 59(4), 510-520.

Lee, A. R. (1999) Application of modified fuzzy AHP method to analyze bolting sequence of structural joints, UMI Dissertation Services, A Bell \& Howell Company. 
Leung, S. C. H., Tsang, S. O. S., Ng, W. L. and Wu, Y. (2007) A robust optimization model for multi-site production planning problem in an uncertain environment, European Journal of Operational Research, 181, 224-238.

Liang, T.-F. (2011) Application of fuzzy sets to manufacturing/distribution planning decisions in supply chains, Information Sciences, 181, 842-854.

Pan, N.-F. (2008) Fuzzy AHP approach for selecting the suitable bridge construction method, Automation in Construction, 17, 958-965.

Peidro, D., Mula, J., Poler, R. and Lario, F.-C. (2009) Quantitative models for supply chain planning under uncertainty: a review, International Journal of Advanced Manufacturing Technology, 43, 400-420.

Pishvaee, M. S. and Torabi, S. A. (2010) A possibilistic programming approach for closed-loop supply chain network design under uncertainty, Fuzzy Sets and Systems, 161, 2668-2683.

Selim, H., Araz, C. and Ozkarahan, I. (2008) Collaborative production-distribution planning in supply chain: a fuzzy goal programming approach, Transportation Research Part E: Logistics and Transportation Review, 44 (3), 396-419.

Sen, S. and Higle, J. L. (1999) An introductory tutorial on stochastic linear programming models, Interfaces, 29(2), 33-61.

Shin, H. J. and $\mathrm{Ru}$, J. P. (2010) Robust production and transportation planning for TFT-LCD industry under demand and price uncertainties using scenario model, Journal of the Korea Academia-Industrial Cooperation Society, 11(9), 3304-3310.

Torabi, S. A. and Hassini, E. (2009) Multi-site production planning integrating procurement and distribution plans in multi-echelon supply chains: an interactive fuzzy goal programming approach, International Journal of Production Research, 47(19), 5475-5499.

Vahidnia, M., Alesheikh, A. A. and Alimohammadi, A. (2009) Hospital site selection using fuzzy AHP and its derivatives, Journal of Environmental Management, 90, 3048-3056. 
Wang, J. T. and Shu, Y. F. (2005) Fuzzy decision modelling for supply chain management, Fuzzy Sets and Systems, 150, 107-127.

Zhang, X., Prajapati, M. and Peden, E. (2011) A stochastic production planning model under uncertain seasonal demand and market growth, International Journal of Production Research, 49(7), 1957-1975.

Zimmermann, H. J. (1976) Description and optimization of fuzzy systems, International Journal of General Systems, 2(4), 209-215.

Zimmermann, H. J. (1978) Fuzzy programming and linear programming with several objective functions, Fuzzy Sets and Systems, 1, 45-55. 